\title{
PERFIL DAS DEMANDAS JUDICIAIS POR MEDICAMENTOS EM MUNICÍPIOS DO ESTADO DA BAHIA
}

\author{
Joslene Lacerda Barreto ${ }^{a}$ \\ Marcelo Tavares Pereira ${ }^{b}$ \\ Maria do Carmo Lessa Guimarãesc \\ Thais Rodrigues Penaforte ${ }^{d}$ \\ Vera Lúcia Almeida Formiglie
}

\section{Resumo}

Este estudo objetivou descrever o perfil das demandas judiciais por medicamentos em municípios do Estado da Bahia, de 2006 a 2010, agregando conhecimento empírico sobre o processo de "judicialização" da saúde, em relação à garantia do acesso a medicamentos. Trata-se de um estudo descritivo baseado em dados secundários dos arquivos das Secretarias Municipais de Saúde referentes às ações judiciais movidas por munícipes. Os resultados revelam que foi impetrado no período um total de 228 ações solicitando 574 medicamentos. Observa-se também que faltam informações relevantes, como relatórios médicos, na maioria das ações. Em três municípios, a maioria dos medicamentos solicitados não consta em nenhum elenco de programas da Assistência Farmacêutica (AF) do Sistema Único de Saúde (SUS); em um município, 50\% dos medicamentos solicitados é do componente básico. Prevalecem solicitações de medicamentos das classes sistema cardiovascular, aparelho digestivo e metabolismo e sistema nervoso central, segundo a classificação Anatomical Therapeutic Chemical (ATC). Conclui-se que, nas Secretarias Municipais de Saúde, a área técnica responsável pelo uso racional de medicamentos é reduzida a fornecedora destes insumos e não há uma sistemática de comunicação e parceria entre esta e o judiciário que viabilize a tomada de decisão fundamentada, minimizando riscos para o paciente e melhorando a qualidade da assistência à saúde.

Palavras-chave: Assistência farmacêutica. Acesso a medicamentos. Ações judiciais.

\footnotetext{
aFarmacêutica integrante do Núcleo de Estudos e Pesquisa em Assistência Farmacêutica da Faculdade de Farmácia e doutoranda em Administração do Programa de Pós-Graduação em Administração da Universidade Federal da Bahia - UFBA - Salvador (BA), Brasil. ${ }^{\text {b} F a r m a c e ̂ u t i c o ~ i n t e g r a n t e ~ d o ~ N u ́ c l e o ~ d e ~ E s t u d o s ~ e ~ P e s q u i s a ~ e m ~ A s s i s t e ̂ n c i a ~ F a r m a c e ̂ u t i c a ~ d a ~ F a c u l d a d e ~ d e ~ F a r m a ́ c i a ~-~ U F B A ~-~}$ Salvador (BA), Brasil.

'Professora Associada III da Faculdade de Farmácia e Professora Permanente do Núcleo de Pós Graduação em Administração UFBA - Salvador (BA), Brasil.

dProfessora Adjunta Il da Faculdade de Farmácia - UFBA - Salvador (BA), Brasil.

eProfessora Adjunta IV da Faculdade de Medicina - UFBA - Salvador (BA), Brasil.

Endereço para correspondência: Núcleo de Estudos e Pesquisas em Assistência Farmacêutica (NEPAF) - Rua Barão do Jeremoabo, 147, Ondina - Salvador (BA),Brasil - CEP: 40.170-115 - E-mail: nepaf.ufba@gmail.com
} 
Revista Baiana de Saúde Pública
PROFILE OF THE JUDICIAL DEMANDS CONCERNING MEDICINES

IN THE MUNICIPALITIES OF BAHIA

\begin{abstract}
The research aimed to describe the profile of the judicial demands concerning medicines in the municipalities in the State of Bahia from 2006 to 2010, adding practical knowledge regarding the process of jurisdiction of the department of health in relation to the guaranteed access to medicines. It is a descriptive study based on data file at the Municipal Health Departments, referring to judicial demands made by locals. The results revealed that 228 actions soliciting 574 medicines were registered in the period. A lack of relevant information, such as medical reports, was observed in the majority of actions. In three of the municipalities the majority of medicines requested are not listed in any index of the Unified Health System (UHS) Pharmaceutical Assistance (PA) programs; in one municipality, 50\% of the medicines solicited is that of the basic component. Those in classes such as cardiovascular system, digestive system and metabolism and central nervous system prevail according to the Anatomical Therapeutic Chemical (ATC) classification. The results reveal that the technical area responsible for the rational use of medications is reduced to a mere supplier of these inputs and highlights the need for improvements in the management of this sector and a systematic communication and partnership with the judiciary, enabling a more reasoned decision without risk to the patient.
\end{abstract}

Keywords: Pharmaceutical services. Public health administration. Health litigation.

\title{
PERFIL DE LAS DEMANDAS JUDICIALES POR MEDICAMENTOS EN MUNICIPIOS DEL ESTADO DE BAHIA
}

\section{Resumen}

Este estudio objetivó describir el perfil de las demandas judiciales por medicamentos en municipios del Estado de Bahía, de 2006 a 2010, agregando conocimiento empírico sobre el proceso de judicialización de la salud, en cuanto a la garantía del acceso a medicamentos. Se trata de un estudio descriptivo basado en datos secundarios de los archivos de las Secretarias Municipales de Salud, referentes a las acciones judiciales movidas por munícipes. Los resultados revelan que fueron requeridos en el período un total de 228 acciones solicitando 574 medicamentos. Fue observado también que faltan informaciones relevantes como informes 
médicos en la mayoría de las acciones. En tres de los municipios, la mayoría de los medicamentos solicitados no constan en ninguna lista de programas de Asistencia Farmacéutica (AF) del Sistema Único de Salud (SUS); en un municipio, 50\% de los medicamentos solicitados es del componente básico. Prevalecen solicitaciones de medicamentos de las clases sistema cardiovascular, aparato digestivo y metabolismo y sistema nervioso central según la clasificación Anatomical Therapeutic Chemical (ATC). Se concluye que, en las Secretarias Municipales de Salud, el área técnica responsable del uso racional de medicamentos está reducida a mero proveedor de estos insumos y no hay una sistemática de comunicación y colaboración entre ella y el sistema judicial, que viabilice la toma de decisiones mejor basada, minimizando riesgos para el paciente y mejorando la calidad de la asistencia a la salud.

Palabras-clave: Asistencia farmacéutica. Acceso a medicamentos. Acciones judiciales.

\section{INTRODUÇÃO}

A Constituição Federal Brasileira estabelece, no seu artigo 196, a saúde como um direito de todos e dever do Estado. Tal direito é garantido por políticas sociais e econômicas. A Lei no 8080/90, ao regulamentar o conjunto das ações e serviços de saúde no país, inclui como campo de atuação do Sistema Único de Saúde (SUS) a execução de ações de assistência terapêutica integral, inclusive farmacêutica, e a formulação da política de medicamentos. ${ }^{1}$

Uma das primeiras orientações do setor foi explicitada com a Portaria $\mathrm{n}^{\circ}$ 3916/98, que definiu a Política Nacional de Medicamentos (PNM) e o estabelecimento de diretrizes que assegurem o acesso da população a medicamentos seguros, eficazes e de qualidade, com menor custo, a partir de um esforço compartilhado entre as três esferas do governo para o alcance deste propósito. A PNM também define as prioridades para implementação da área de medicamentos, referendando mais uma vez o direito à Assistência Farmacêutica (AF) e todas as suas atividades relacionadas. ${ }^{2}$

No âmbito do SUS, as ações relacionadas à AF foram oficializadas por meio da Resolução no 338/04, que estabeleceu seus princípios norteadores e eixos estratégicos de atuação, objetivando não apenas a ampliação do acesso, mas principalmente a promoção do Uso Racional de Medicamentos (URM) e insumos para a saúde. ${ }^{3}$ Esta organização política da AF também teve como objetivo aperfeiçoar o seu processo de descentralização, reorganizando-a em três componentes: básico, especializado e estratégico. 
Revista Baiana de Saúde Pública medicamentos e insumos essenciais, destinados ao atendimento de agravos prevalentes e prioritários da Atenção Básica. O componente especializado é considerado uma estratégia para garantia do acesso a medicamentos excepcionais, definidos a partir de critérios de diagnóstico, indicação, tratamento e outros parâmetros constantes nos Protocolos Clínicos e Diretrizes Terapêuticas. O componente estratégico inclui os medicamentos utilizados para o tratamento de agravos específicos, contemplados em programas coordenados pelo Ministério da Saúde, de caráter transmissível e/ou de alto impacto na saúde da população, como o programa DST/AIDS, Tuberculose, Hanseníase e outras endemias focais. ${ }^{4}$

Apesar de todo este arranjo operacional e político, não se pode dizer que a AF no SUS esteja plenamente consolidada. Segundo Barreto e Guimarães, ${ }^{5}$ observa-se um grande distanciamento entre o disposto na legislação da AF e o que efetivamente vem sendo compreendido e exercido pelos gestores e farmacêuticos. Estudos revelam que as ações e atividades da AF prestada nos municípios brasileiros ainda são insuficientes para garantir o acesso e o uso racional dos medicamentos, ${ }^{6,7}$ apresentando debilidades na infraestrutura, na operacionalidade dos serviços e no atendimento da demanda da população por medicamentos. ${ }^{8}$

Esta demanda reprimida vem encontrando na via judicial a alternativa para garantia do acesso a medicamentos no SUS, que teve início na década de 1990, com solicitações de antirretrovirais para tratamento do HIV/AIDS. A partir daí, esta via de acesso vem crescendo anualmente e passou a ser alvo de discussões tanto na área de saúde como na área jurídica, com repercussões na organização dos serviços dessas áreas.

Tentar identificar fatores determinantes para esse aumento na demanda das ações judiciais no Brasil é uma tarefa complexa, ao considerar as realidades social, econômica e política do país. De acordo com Macedo et al. ${ }^{9}$, alguns fatores contribuem para o aumento das solicitações judiciais de medicamentos do componente especializado da AF, tais como: a desatualização e dificuldade de interpretação dos protocolos, a burocratização dos serviços farmacêuticos, a dispensação centralizada e a inadequação da prescrição aos protocolos clínicos e terapêuticos.

O Conselho Nacional de Secretários de Saúde (CONASS) destaca ainda o processo de incorporação tecnológica na área da saúde, intensificado nas últimas décadas, o qual resultou no aumento das demandas da sociedade, que exerce pressões junto aos setores responsáveis pela oferta dessas novas tecnologias. ${ }^{10}$ Além disso, tem sido também apontada a pressão das indústrias farmacêuticas quanto aos prescritores, 
por meio, principalmente, de estratégias de marketing, para que os produtos lançados no mercado sejam incorporados ao elenco do SUS..$^{11,12}$

Com relação à atuação do Poder Judiciário no acesso de pacientes aos medicamentos, a literatura sobre o tema discute que esta participação pode reduzir violações de direito cometidas pelo Estado contra seus cidadãos, que possuem um direito assegurado de exigir essa obrigação estatal; por outro lado, esta influência não pautada em concepções técnicas qualificadas pode, além de propiciar práticas terapêuticas irracionais, contradizendo um objetivo precípuo das políticas farmacêuticas, desorganizar o orçamento e a administração da AF.9,13

Um estudo realizado em Santa Catarina revela que a concretização do direito de acesso à saúde por meio do acesso a medicamentos via judicial não está promovendo, necessariamente, a conscientização deste direito pelo usuário, que atribui o êxito no resultado do processo ao médico, ao advogado ou ao político que o ajudou a conseguir o medicamento e, impotente diante do poder público, não sente segurança quanto à continuidade do benefício. ${ }^{14}$

No Estado da Bahia, um estudo analisou as ações judiciais para o acesso a medicamentos excepcionais no ano de 2003, constatando que naquele ano ocorreram 19 ações judiciais e 5 solicitações formalizadas por pacientes à Secretaria Estadual de Saúde, pleiteando produtos considerados excepcionais. ${ }^{15}$ Outro trabalho identificou, no município de Salvador (BA), 264 pleitos de medicamentos por meio de processos administrativos e judiciais em 2006, sendo este total significativamente maior que nos anos anteriores: 2002 (1), 2003 (14), 2004 (87) e 2005 (166). ${ }^{16}$

Diante deste contexto, este estudo objetivou descrever o perfil das demandas judiciais por medicamentos solicitadas às Secretarias de Saúde de municípios do Estado da Bahia, no período de 2006 a 2010, visando contribuir para agregar conhecimento empírico sobre o processo denominado de "judicialização" da saúde, considerando a garantia do acesso a medicamentos pela população e o princípio constitucional do direito à saúde.

\section{MATERIAL E MÉTODOS}

Foram estudados quatro municípios pertencentes a diferentes macrorregiões de saúde ${ }^{17}$ entre os dez maiores do Estado da Bahia: Salvador, Feira de Santana, Vitória da Conquista e Ilhéus. Os três maiores municípios foram escolhidos por representarem mais de $25 \%$ da população total do Estado e o quarto caso foi sorteado entre os restantes. 
Revista Baiana de Saúde Pública
Definiu-se como universo da pesquisa as coordenações da AF dos municípios estudados. A unidade de análise foram as demandas por medicamentos por meio de ações judiciais movidas por munícipes contra as respectivas Secretarias Municipais de Saúde, no período de janeiro de 2006 a dezembro de 2010. As demandas por medicamentos oriundas de processos administrativos, tanto as encaminhadas diretamente pelo paciente ou seu representante como as mediadas pelo Ministério e Defensoria Pública, não foram incluídas neste estudo.

Trata-se de uma pesquisa de natureza descritiva baseada na análise de dados secundários extraídos dos documentos encaminhados pelas respectivas Procuradorias Jurídicas municipais às coordenações da AF das Secretarias de Saúde dos municípios selecionados, para cumprimento de ações judiciais geradas pelas demandas de pacientes por medicamentos. Foram também consultados os bancos de dados eletrônicos construídos por algumas destas secretarias para acompanhar tais demandas.

O levantamento dos dados relativos às demandas por medicamentos por via judicial foi feito apenas nos arquivos das Secretarias Municipais de Saúde e não incluiu dados dos órgãos do Judiciário, porque, até o final do trabalho de campo, o Tribunal de Justiça da Bahia não disponibilizava estes dados de forma informatizada e/ou sistematizada de modo que pudesse viabilizar o levantamento e a análise das informações pretendidas.

Para a análise do perfil das demandas por medicamentos por meio de ações judiciais foram selecionados dados relativos a:

a) Número total por ano das demandas por medicamentos por via judicial, unidade de saúde de origem da prescrição, existência de relatório médico, nome e endereço do paciente demandante da ação;

b) Condutor da ação judicial (Defensoria e Ministério Público, advogados particulares);

c) Existência de registro do medicamento solicitado na Agência Nacional de Vigilância Sanitária (ANVISA) na data da sua solicitação;

d) Classe terapêutica segundo a Anatomical Therapeutic Chemical Classification (ATC) da Organização Mundial da Saúde (OMS);

e) Pertencimento dos medicamentos solicitados a um dos programas de AF do SUS no período da solicitação.

Foi utilizado para a análise o programa MS Excel 2003 ${ }^{\circledR}$, o qual possibilitou a sistematização dos dados, seu processamento, tabulação e construção de gráficos e tabelas. 
O projeto foi avaliado e aprovado pelo Comitê de Ética em Pesquisa do Hospital Universitário Prof. Edgar Santos, conforme as normas previstas na Resolução n 196/06 do Conselho Nacional de Saúde (Projeto n 262/07). Os dados utilizados não permitem, de nenhuma forma, a identificação nominal dos sujeitos demandantes das ações judiciais.

\section{RESULTADOS}

Observou-se que, no período de 2006 a 2010, foi impetrado contra os municípios pesquisados um total de 228 ações judiciais solicitando 574 medicamentos, perfazendo uma média de 2,5 medicamentos por ação judicial.

Observa-se que em 2006 o número de pleitos por medicamentos por meio de ações judiciais foi pequeno. O município que apresentou o maior número foi Ilhéus, com seis ocorrências, Salvador com três e Feira de Santana com apenas duas ocorrências. No município de Vitória da Conquista não há registro de ações judiciais anteriores ao ano de 2008.

Não foi observada uniformidade na tendência de crescimento das ações nos diferentes municípios. No entanto, chama a atenção o expressivo aumento no número de ações judiciais ocorrido em Salvador e Ilhéus no ano de 2008 e em Vitória da Conquista em 2009, conforme o Gráfico 1.

Foi constatado que em 57\% das 228 ações encontradas o autor/paciente buscou o Ministério ou Defensoria Pública para iniciar o processo de petição judicial do tratamento medicamentoso, enquanto apenas 7\% o fizeram através de advogados contratados.

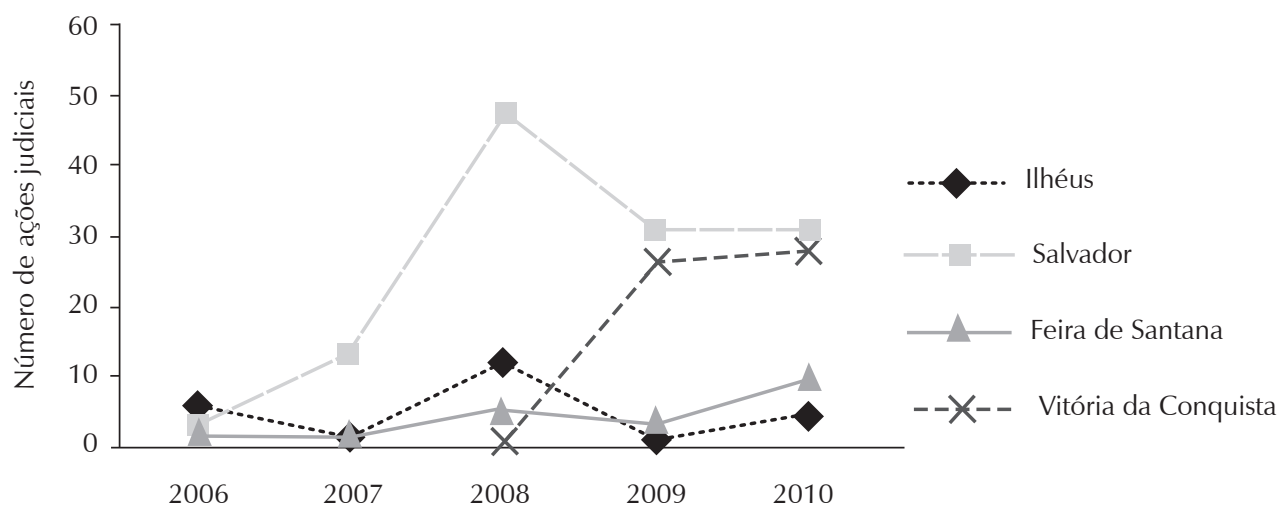

Gráfico 1 - Universo das ações judiciais impetradas contra municípios da Bahia, Brasil - 2006 a 2010 
Revista Baiana de Saúde Pública
Entretanto, observa-se que em $36 \%$ do total de demandas judiciais por medicamentos, nos quatro municípios estudados, não constavam essa informação na documentação encaminhada às coordenações da AF, destacando-se Salvador, com 57\%.

No Gráfico 2 é possível observar que em Feira de Santana todos os documentos relativos às demandas judiciais armazenados na coordenação da AF continham informação sobre a origem da prescrição médica, demonstrando que 57\% delas foram provenientes de estabelecimentos públicos. Em Salvador e Vitória da Conquista esta informação estava ausente em mais da metade dos documentos e em Ilhéus, em 38\%.

A grande maioria das demandas judiciais, nos casos estudados, foi atendida pela gestão municipal, apesar de outras esferas figurarem como réus nos processos. É possível constatar que Salvador foi a exceção, pois 49\% das solicitações foram cumpridas pelo Estado, $35 \%$ pelo município, $2 \%$ pela União, e em $13 \%$ das ações não foi possível identificar a esfera de governo que atendeu à solicitação.

Um achado que chamou a atenção foi o fato de não constarem relatórios médicos na maioria dos documentos arquivados nas coordenações da AF de Salvador (67\%) e de Vitória da Conquista (64\%), e em quase metade das demandas de Ilhéus (42\%) e Feira de Santana (48\%). O relatório médico é um documento importante para subsidiar a decisão sobre o medicamento solicitado.

Dos 574 medicamentos solicitados judicialmente, 7 (1,2\%) não estão registrados na ANVISA, sendo que 5 deles foram atendidos pelo município ou pelo Estado.

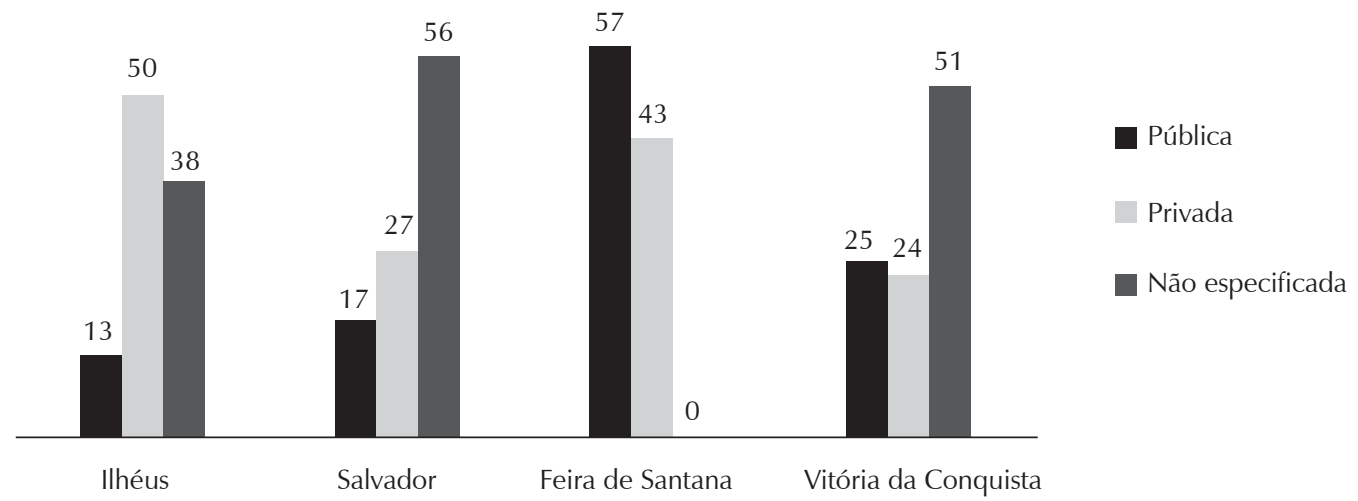

Gráfico 2 - Origem do atendimento de pacientes autores de demandas judiciais contra municípios do estado da Bahia, Brasil, entre 2006 e 2010 
Os dados apresentados no Gráfico 3 revelam que a maioria dos medicamentos solicitados nos municípios de Salvador, Feira de Santana e Vitória da Conquista não consta em nenhum elenco dos programas oficiais da AF do SUS. Dentre os incluídos nos elencos dos programas oficiais, prevalecem as solicitações de medicamentos do componente básico da AF. Já no município de Ilhéus prevaleceram demandas por medicamentos constantes dos programas oficiais, sendo mais de $50 \%$ deles pertencentes ao componente básico da AF.

Cabe ressaltar que alguns medicamentos não pertencentes aos elencos vigentes dos programas oficiais da AF foram incluídos em revisões posteriores aos pleitos. Estão, neste caso, o atenolol, incluído na relação do Componente Básico em 2008, a clonidina, o clopidogrel, o diltiazen e a isossorbida, na atualização de 2009.

Observa-se na Tabela 1 que, nos quatro municípios, a maioria dos medicamentos solicitados pertence ao sistema cardiovascular, aparelho digestivo e metabolismo e sistema nervoso central, segundo classificação pelo primeiro nível do ATC (Ilhéus: 93,3\%; Salvador: 61,7\%; Feira de Santana: 93,3\%; Vitória da Conquista: 85,5\%). Destaca-se ainda o grande número de solicitações de medicamentos do sistema respiratório (tratamento da asma) em Vitória da Conquista e Feira de Santana e de medicamentos antineoplásicos e agentes moduladores do sistema imunológico em Salvador.

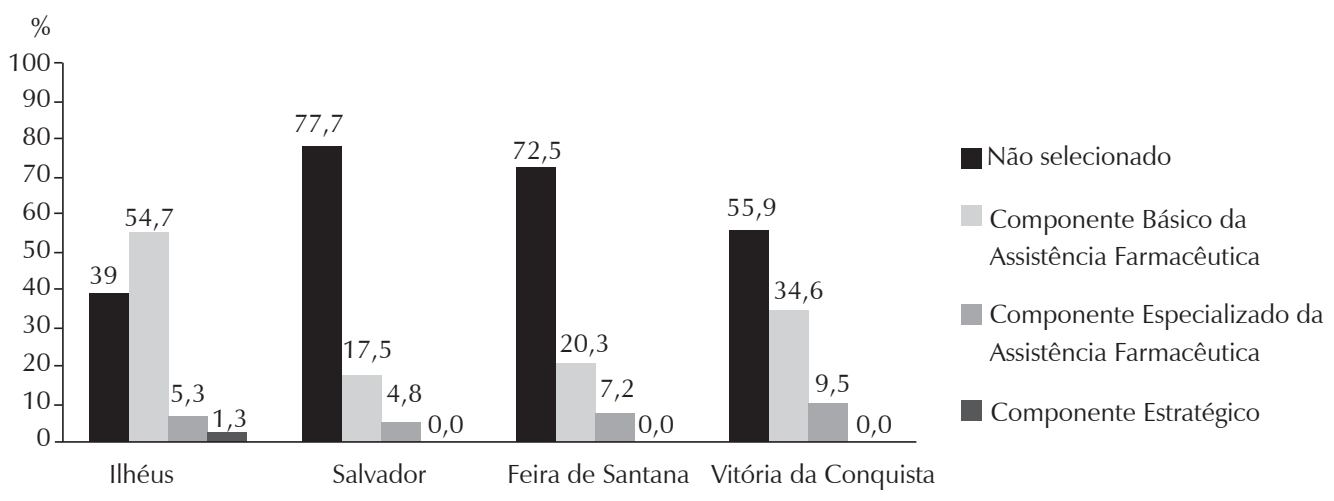

Gráfico 3 - Distribuição dos medicamentos solicitados por Programas de Assistência Farmacêutica do Sistema Único de Saúde em municípios baianos - 2006 e 2010 
Revista Baiana de Saúde Pública
Tabela 1 - Distribuição dos medicamentos solicitados, segundo classificação pelo primeiro nível da Anatomical Therapeutic Chemical Classificacio (ATC) em munípios da Bahia- Brasil. 2006-2010

\begin{tabular}{|c|c|c|c|c|c|c|c|c|}
\hline Primeiro nível da ATC & Salvador & $\%$ & Ilheus & $\%$ & $\begin{array}{l}\text { Vitoria da } \\
\text { Conquista }\end{array}$ & $\%$ & $\begin{array}{l}\text { Feira de } \\
\text { Santana }\end{array}$ & $\%$ \\
\hline C. Sistema cardiovascular & 73 & 29,1 & 28 & 37,3 & 50 & 27,9 & 17 & 24,6 \\
\hline $\begin{array}{l}\text { L. Antineoplasicos e } \\
\text { agentes moduladores do } \\
\text { sistema imunológico }\end{array}$ & 49 & 19,5 & 2 & 2,7 & 5 & 2,8 & 2 & 2,9 \\
\hline $\begin{array}{l}\text { A. Aparelho digestivo e } \\
\text { metabolismo }\end{array}$ & 41 & 16,3 & 34 & 45,3 & 38 & 21,2 & 20 & 29,0 \\
\hline N. Sistema nervoso central & 41 & 16,3 & 8 & 10,7 & 38 & 21,2 & 22 & 31,9 \\
\hline $\begin{array}{l}\text { G. Aparelho genito- } \\
\text { urinário e hormônios } \\
\text { sexuais }\end{array}$ & 9 & 3,6 & & 0 & 5 & 2,8 & & 0,0 \\
\hline S. Orgãos do sentido & 8 & 3,2 & & 0 & 10 & 5,6 & 2 & 2,9 \\
\hline R. Sistema respiratório & 7 & 2,8 & & 0 & 19 & 10,6 & 4 & 5,8 \\
\hline $\begin{array}{l}\text { M. Sistema músculo- } \\
\text { esquelético }\end{array}$ & 6 & 2,4 & & 0 & 5 & 2,8 & 1 & 1,4 \\
\hline $\begin{array}{l}\text { J. Antiinfeciosos gerais para } \\
\text { uso sistêmico }\end{array}$ & 5 & 2,0 & & 0 & 2 & 1,1 & 1 & 1,4 \\
\hline $\begin{array}{l}\text { B. Sangue e orgãos } \\
\text { hematopoiéticos }\end{array}$ & 4 & 1,6 & & 0 & 2 & 1,1 & & 0,0 \\
\hline $\begin{array}{l}\text { D. Medicamentos } \\
\text { dermatológicos }\end{array}$ & 4 & 1,6 & & 0 & 1 & 0,6 & & 0,0 \\
\hline $\begin{array}{l}\text { H. Preparados hormonais } \\
\text { sistêmicos, exc. } \\
\text { Hormonios sexuais }\end{array}$ & 2 & 0,8 & 3 & 4 & 4 & 2,2 & & 0,0 \\
\hline V. Vários & 2 & 0,8 & & 0 & & 0,0 & & 0,0 \\
\hline Total & 251 & 100,0 & 75 & 100 & 179 & 100 & 69 & 100 \\
\hline
\end{tabular}

\section{DISCUSSÃO}

A primeira constatação do estudo foi a de que houve aumento no número de ações judiciais em todos os municípios analisados, entre os anos de 2006 e 2010, o que confirma os resultados de outros estudos realizados em vários Estados e municípios do Brasil para produtos selecionados ou não pelos programas do SUS.

Os dados obtidos na caracterização das demandas judiciais por medicamentos nos municípios estudados nesta pesquisa levantam questionamentos tanto em relação à organização dos serviços quanto no que diz respeito às correlações de forças que estão dadas na sociedade quando se trata de acesso e direito aos serviços públicos.

Nesse sentido, é importante também reconhecer que essa demanda por medicamentos por meio das ações judiciais revela uma nova forma da população intervir na gestão do SUS, quando traz à tona as necessidades epidemiológicas não prevalentes na sociedade, não previstas na rede, como foi o caso em Salvador, onde $74 \%$ de solicitações judiciais de medicamentos não constavam dos programas oficiais da AF. 
Por outro lado, o fornecimento pode significar o direito de uma parcela da população escolher a sua terapia, enquanto outra enorme parcela não tem oportunidade de escolha, principalmente por falta de informação, limitando-se aos medicamentos que constam das listas oficiais do SUS. A maioria dos estudos sobre esta questão tem concluído, além disso, que essas solicitações prejudicam a padronização de medicamentos e oneram o sistema, constituindo-se numa demanda não esperada para os gestores.

É possível levantar, a partir da análise dos tipos de medicamentos solicitados, da literatura e do conhecimento sobre a assistência à saúde no Estado da Bahia, algumas suposições sobre prováveis situações motivadoras da escolha da via judicial por parte de pacientes para garantir sua terapêutica, embora o desenho desta pesquisa não tenha contemplado a investigação das causas que motivaram o aumento dessa busca:

i) o desabastecimento de medicamentos na rede do SUS nos municípios;

ii) o fato de a patologia não estar contemplada no Protocolo de Tratamento do Programa de Medicamentos Excepcionais;

iii) maior consciência do direito à saúde integral por parte dos pacientes;

iv) altos preços dos medicamentos;

v) ausência de informação na rede SUS sobre tratamentos medicamentosos disponibilizados;

vi) maior estruturação do judiciário e atuação das promotorias/defensorias públicas nos últimos anos; e

vii) desatualização das listas dos programas oficiais do SUS, em especial a oncologia. Vale aqui ressaltar que a Relação Municipal de Medicamentos Essenciais (REMUME) de Salvador só foi revisada no ano de 2010, último ano da série temporal deste estudo, o que também pode sugerir a necessidade sentida pela população de novos medicamentos não dispensados na rede pública.

O fato de a condução dos processos judiciais ter sido feita, na sua maioria, nos municípios do interior, pelo Ministério ou Defensoria Pública, pode indicar maior acesso da população a esses órgãos no interior do Estado e diálogo mais próximo e frequente entre essas instâncias jurídicas e o Setor de Saúde Municipal.

O cumprimento das demandas judiciais foi diferenciado na capital e demais municípios. Nestes últimos, os pleitos foram cumpridos, em sua maioria, pela própria instância municipal, e em Salvador pelo nível estadual do SUS, uma vez que os tipos de 
Revista Baiana de Saúde Pública medicamentos objetos da ação judicial que prevaleceram neste município estão no rol dos mais especializados e que ainda estão sob a responsabilidade financeira do Estado e da União.

Os dados levantados, confirmando os resultados de outros estudos ${ }^{18-20}$, revelam uma acentuada insuficiência de informações tanto nas documentações relativas às ações judiciais arquivada nas coordenações da AF dos municípios estudados quanto no banco de dados eletrônico de um dos municípios. Esta situação, além de comprometer a análise técnica e possíveis esclarecimentos necessários ao cumprimento dos pleitos pelo setor de AF, repercutiu também na pesquisa, dificultando e até impossibilitando, de certa forma, uma análise mais aprofundada, incluindo as razões que geraram a ação judicial, pela ausência de dados sobre o endereço e/ou contato da maioria dos pacientes e de prescritores.

Esta insuficiência de informações no âmbito das Secretarias Municipais de Saúde é potencializada pela restrição do acesso ao sistema informatizado dos Tribunais de Justiça na Bahia, o que dificulta o monitoramento dos processos, não só por pesquisadores como pela própria população. Esta situação evidencia problemas na comunicação entre a área judicial, que demanda os medicamentos, e a área técnica, responsável pela dispensação dos medicamentos solicitados pelos pacientes.

Neste estudo, observou-se que a decisão do juiz em determinar o fornecimento do medicamento por meio de liminar é respaldada na existência de relatório e/ou prescrição médica nos autos do processo, embora estas informações, como foi relatado, não constem da maioria das solicitações arquivadas na área técnica da AF municipal. Este achado é recorrente em outras pesquisas, ${ }^{13,21}$ fortalecendo a ideia de que estes documentos se constituem, para os juízes, em provas suficientes para respaldar suas decisões, legitimadas pelo argumento do direito inviolável à saúde, embora nem sempre de acordo com o disposto nas políticas de saúde e no orçamento público. É possível concordar com Pepe, ${ }^{22}$ quando afirma que a falta de conhecimento técnico dos juízes sobre o fornecimento de medicamentos leva, na grande maioria dos casos, a decisões fundamentadas apenas na urgência dos pedidos e no dever do Estado em fornecê-los.

Macedo et al. ${ }^{9}$ alertam para a importância da análise técnica subsidiando a tomada de decisão para o fornecimento de medicamentos pela via judicial, principalmente em relação aos medicamentos não disponíveis nos programas oficiais da AF no SUS. A solicitação, nestes casos, pode ter sido provocada pelos limites da essencialidade que norteia a seleção de medicamentos, impondo restrições às listas oficiais e até deixando de oferecer alternativas terapêuticas. Esta análise técnica precisa considerar, além de critérios 
relacionados à política e aos serviços de saúde, as necessidades individualizadas, afinal as listas de medicamentos devem acompanhar os avanços tecnológicos e sociais.

Machado et al. ${ }^{18}$ também chamam a atenção para $\mathrm{o}$ fato de que $\mathrm{o}$ atendimento de demandas judiciais sem a devida análise técnica, inclusive quanto à existência de alternativas terapêuticas disponíveis, estabelecidas em elencos padronizados ou recomendados em protocolos clínicos e diretrizes terapêuticas, promove desarticulação das ações e serviços da AF, estimula a medicalização e se constitui num obstáculo ao URM. $\mathrm{Na}$ busca pela garantia do direito à saúde, o mesmo vem sendo reduzido ao fornecimento do medicamento, tendo a prescrição médica como única prova processual, o que não é suficiente para garantir o fornecimento responsável de medicamentos seguros. ${ }^{13}$

O alto percentual de demandas judiciais por medicamentos não incluídos nas listas oficiais, encontrado em três municípios deste estudo, reforça uma tendência a mudança no perfil das ações judiciais, observada também em pesquisas recentes realizadas em Minas Gerais, ${ }^{18}$ Rio de Janeiro ${ }^{13}$ e São Paulo, ${ }^{9,19}$ em contraponto a estudos anteriores que apontavam a maior parte dos medicamentos solicitados pertencentes às listas oficiais. ${ }^{23-25}$

Embora as decisões judiciais para o fornecimento de medicamentos não incluídos na seleção dos programas oficiais da AF do SUS estejam balizadas pelo princípio defensável do direito à saúde, essa via de acesso não exclui a necessidade de observância de certos critérios técnicos, tais como: existência de alternativas terapêuticas adequadas ao caso clínico do paciente demandante, existência de registro do medicamento no país, indicação para a patologia apresentada pelo paciente, além da análise de comparação de custo em relação às farmacoterapias similares disponíveis. Por estas razões, o Conselho Nacional de Justiça recomendou, em 2010, que os Tribunais de Justiça constituam apoio técnico formado por médicos e farmacêuticos, visando auxiliar o juiz na tomada de decisão que envolva medicamentos e outras demandas de saúde. ${ }^{9}$

Por outro lado, chama a atenção a demanda por medicamentos constantes das listas oficiais, especialmente do componente básico da AF, que representa mais de $50 \%$ das solicitações judiciais em um dos municípios. Estas demandas, segundo Vieira, ${ }^{26}$ podem ser consideradas pertinentes, uma vez que os medicamentos deveriam estar disponíveis aos pacientes na rede do SUS. Este achado pode revelar tanto a provável existência de problemas relacionados à gestão desses programas como também o desconhecimento ou a não adesão dos prescritores às listas oficiais e, especificamente para os medicamentos do componente especializado, pode revelar uma fuga à burocracia do referido programa, como referem Sant'Ana et al. ${ }^{13}$ Tais autores consideram que as referidas demandas podem até cooperar para 
Revista Baiana de Saúde Pública promoção do uso racional do medicamento, desde que estes pleitos sejam analisados sob a perspectiva técnica, garantindo que o tratamento solicitado seja adequado às condições de saúde do paciente solicitante e às políticas públicas instituídas.

Este estudo também confirma o achado de outras pesquisas ${ }^{18,19,22}$ em relação à maior frequência de solicitações de medicamentos incluídos nas classes $\mathrm{C}, \mathrm{L}, \mathrm{A}$ e $\mathrm{N}$ da ATC, o que indica a necessidade de maior atenção da gestão da AF para esses grupos de medicamentos e as respectivas patologias para as quais são indicados.

\section{CONSIDERAÇÕES FINAIS}

Os achados deste estudo apontam a necessidade de constantes revisões nas políticas públicas de $\mathrm{AF}$, em relação à gestão, ao financiamento, à inclusão de novas patologias, atualização de protocolos clínicos e revisão dos elencos, visando à incorporação de novas tecnologias no SUS para atender às necessidades epidemiológicas da população.

Fornecer medicamentos que não constam nas relações padronizadas oficiais por via judicial, situação da maioria dos medicamentos solicitados neste estudo, remete a uma discussão sobre a abrangência e a competência técnica envolvidas na avaliação destes pleitos, e pode ter diferentes sentidos, servindo como indicador da defasagem da política e como um alerta para a necessidade de incorporação de novas tecnologias farmacêuticas, de atualização sistemática das listas de medicamentos diante das inovações existentes, sua divulgação e adesão por parte dos prescritores.

Contudo, é significativo o achado de que muitos dos medicamentos solicitados por meio das ações judiciais deveriam estar disponíveis à população na rede SUS, nas Unidades Básicas de Saúde (UBSs) ou nas unidades de referência através dos programas oficiais da AF. Tais resultados suscitam reflexões sobre as razões que motivaram aqueles pacientes a buscarem a via judicial como meio de acesso à sua terapêutica, indicando fragilidades nas gestões da AF municipal e estadual, problemas na gestão dos recursos disponíveis para este fim e na cobertura e acessibilidade desses programas.

A ausência dos relatórios médicos nos processos encaminhados à área técnica mostra o quanto parece ser insignificante a análise técnica realizada por parte dos profissionais de saúde sobre as solicitações que geraram uma decisão judicial. Tal fato reduz o setor responsável pelo uso racional de medicamentos a um mero fornecedor destes insumos. Estes problemas, entre outros aspectos, evidenciam a necessidade de que estas demandas judiciais devem ser encaminhadas às coordenações municipais da AF mais bem instruídas e da instauração de uma sistemática de comunicação entre o setor jurídico e essas coordenações. 
A garantia do acesso a medicamentos e da sua utilização de forma racional deve visar à proteção da saúde da população, disponibilizando a terapêutica mais adequada e segura para atender à situação clínica de cada paciente. Supõe-se, portanto, que não devem existir conflitos entre o Judiciário e a área técnica da $\mathrm{AF}$, considerando que a análise técnica é necessária para se comprovar a adequação da prescrição, a existência de alternativas terapêuticas disponíveis no SUS e, quando for o caso, o acompanhamento dos efeitos do uso do medicamento no paciente.

Por fim, estas considerações não pretendem reduzir em importância a prerrogativa da população de recorrer ao Poder Judiciário para garantir seus direitos, mas enfatizar a necessidade de melhorias na gestão da AF nos municípios e no Estado e na divulgação de informações para a população e a classe médica sobre os programas de AF do SUS. Indicam ainda a necessidade de maior capacitação do Judiciário nessa questão, da existência de um suporte técnico para entender e melhor julgar as questões de saúde, além de uma forte parceria entre este setor e a área técnica da AF municipal que possibilite a tomada de decisão bem fundamentada, minimizando os riscos para o paciente e considerando também o conjunto de normas técnicas que orientam e regulam o funcionamento do SUS.

\section{REFERÊNCIAS}

1. Brasil. Lei n. 8.080. Dispõe sobre as condições para a promoção, proteção e recuperação da saúde, a organização e o funcionamento dos serviços correspondentes e dá outras providências. Brasília, DF: Diário Oficial da União; 1990; 20 de setembro de 1990.

2. Brasil. Ministério da Saúde. Portaria MS n. 3.916, de 30 de outubro de 1998. Aprova a Política Nacional de Medicamentos. Brasília, DF: Diário Oficial da União; 1998: 10 de novembro de 1998.

3. Brasil. Ministério da Saúde. Resolução CNS n. 338, de 6 de maio de 2004. Aprova a Política Nacional de Assistência Farmacêutica. Brasília; 2004.

4. Brasil. Ministério da Saúde. Portaria GM/MS n. 204, de 29 de janeiro de 2007. Regulamenta o financiamento e a transferência dos recursos federais para as ações e os serviços de saúde, na forma de blocos de financiamento, com o respectivo monitoramento e controle. Brasília: Ministério da Saúde; 2007.

5. Barreto JL, Guimarães, MCL. Avaliação da Gestão Descentralizada da Assistência Farmacêutica Básica em Municípios Baianos, Brasil. Cad Saúde Pública. 2010;26(6):1207-20. 
Revista Baiana

de Saúde Pública
6. Guerra Jr. AA, Acúrcio FA, Gomes CAP, Miralles M, Girardi SN, Carvalho $\mathrm{CL}$, et al. Disponibilidade de medicamentos essenciais em duas regiões de Minas Gerais, Brasil. Rev Panam Salud Publica. 2004;15(3):168-75.

7. Fraga FNR. A utilização de um modelo lógico para a reorientação dos serviços farmacêuticos no âmbito municipal [Dissertação]. Porto Alegre (RS): Faculdade de Farmácia, Universidade Federal do Rio Grande do Sul; 2005.

8. Alencar TOS, Nascimento MAA. Assistência Farmacêutica no Programa Saúde da Família: encontros e desencontros do processo de organização. Ciênc Saúde Coletiva 2011;16(9):3939-49.

9. Macedo EI, Lopes LC, Barberato-Filho, S. Análise técnica para a tomada de decisão do fornecimento de medicamentos pela via judicial. Rev Saúde Pública. 2011;45(4):706-13.

10. Brasil. Conselho Nacional de Secretários de Saúde. Assistência Farmacêutica no SUS/Conselho Nacional de Secretários de Saúde. Brasília: CONASS; 2011. 186 p. (Coleção Para Entender a Gestão do SUS 2011, 7, p. 130-52).

11. Brasil. Conselho Nacional de Secretários de Saúde. Assistência Farmacêutica: Medicamentos de Dispensação em Caráter Excepcional. Brasília: CONASS; 2004. 64p. (CONASS Documenta 5).

12. Chieffi AL, Barata RCB. Ações judiciais: estratégia da indústria farmacêutica para introdução de novos medicamentos. Rev Saúde Pública. 2010;44(3):421-9.

13. Sant'Ana JMB, Pepe VLE, Figueiredo TA, Osório-de-Castro CGS, Ventura M. Racionalidade terapêutica: elementos médico-sanitários nas demandas judiciais de medicamentos. Rev Saúde Pública. 2011;45(4):714-21.

14. Leite SN, Mafra AC. Que direito? Trajetórias e percepções de usuários no processo de acesso a medicamentos por mandados judiciais em Santa Catarina. Ciênc Saúde Coletiva. 2010;15(Suppl.1):1665-72.

15. Pereira MT, Pena MJL. O acesso do medicamento e a garantia da assistência integral à saúde: um estudo sobre suas implicações no desenvolvimento do PEMAC no estado da Bahia, 2003 [Monografia]. Salvador (BA): Faculdade de Farmácia, Universidade Federal da Bahia; 2004.

16. Conceição Filho JN, Domingues ASP. Acesso a medicamentos por via judicial e processos administrativos: um estudo sobre seu impacto na gestão da assistência farmacêutica no município de Salvador-BA, 2006 [Monografia] Salvador (BA): Faculdade de Farmácia, Universidade Federal da Bahia; 2006.

17. Bahia. Secretaria da Saúde. Plano Diretor de Regionalização (PDR). Salvador:SESAB; 2007. 
18. Machado MAA, Acúrcio FA, Brandão CMR, Faleiros DR, Guerra Jr AA, Cherchiglia ML, et al. Judicialização do acesso a medicamentos no Estado de Minas Gerais, Brasil. Rev Saúde Pública. 2011;45(3):590-8.

19. Chieffi AL, Barata RB. Judicialização da política pública de assistência farmacêutica e eqüidade. Cad Saúde Pública. 2009;25(8):1839-49.

20. Leite SN, Pereira SMP, Silva P, Nascimento Jr JM, Cordeiro BC, Veber AP. Ações judiciais e demandas administrativas na garantia do direito de acesso a medicamentos em Florianópolis-SC. Rev Direito Sanit. 2009;10(2):13-28.

21. Pepe VLE, Figueiredo TA, Simas L, Osório-de-Castro CGS, Ventura M. A judicialização da saúde e os novos desafios da gestão da assistência farmacêutica. Ciênc Saúde Coletiva. 2010;15(5):2405-14.

22. Pepe VLE, Ventura M, Sant'Ana JMB, Figueiredo TA, Souza VR, Simas $L$, et al. Caracterização de demandas judiciais de fornecimento de medicamentos "essenciais" no Estado do Rio de Janeiro, Brasil. Cad Saúde Pública. 2010;26(3):461-71.

23. Messeder AM, Osorio-de-Castro CGS, Luiza VL. Mandados judiciais como ferramenta para garantia do acesso a medicamentos no setor público: a experiência do Estado do Rio de Janeiro, Brasil. Cad Saúde Pública. 2005;21(2):525-34.

24. Borges DCL, Ugá MAD. Conflitos e impasses da judicialização na obtenção de medicamentos: as decisões de primeira instância nas ações individuais contra o Estado do Rio de Janeiro, Brasil, em 2005. Cad Saúde Pública. 2010:26(1):59-69.

25. Vieira FS, Zucchi F. Distorções causadas pelas ações judiciais à política de medicamentos no Brasil. Rev Saúde Pública. 2007;41(2):214-22.

26. Vieira FS. Ações judiciais e direito à saúde: reflexão sobre a observância aos princípios do SUS. Rev Saúde Pública. 2008;42(2):365-9.

Recebido em 24.05.2013 e aprovado em 06.02.2014. 